\title{
Predicted Radar/Optical Feature Fusion Gains For Target Identification
}

\author{
Bart Kahler \\ General Dynamics \\ WPAFB, OH 45433
}

\author{
Erik Blasch \\ Air Force Research Lab \\ WPAFB, OH 45433
}

\begin{abstract}
Airborne tracking and identification (ID) of high value ground targets is a difficult task impacted by sensor, target, and environmental conditions. Layered sensing, using a combination of standoff and short-range sensors, maintains target track and identification in cluttered environments such as cities or densely vegetated areas through sensor diversity. Data, feature, decision, or information fusion is necessary for high confidence target classification to be achieved using multiple sensors and sensor modalities. Target identification performance is improved by exploiting the extra information gained from independent sensing modalities through information fusion for automatic target recognition (ATR). Increased target ID has been demonstrated using spatial-temporal multi-look sensor fusion and decision level fusion. To further enhance target ID performance and increase decision confidence, feature level fusion techniques are being investigated. A fusion performance model for feature level fusion was applied to a combination of sensor types and features to provide estimates of a fusion gain. This paper presents a fusion performance gain for Synthetic Aperture Radar (SAR), electro-optical (EO), and infrared (IR) video stationary target identification.
\end{abstract}

Keywords: decision level fusion, feature level fusion, electro-optical (EO), infrared (IR), synthetic aperture radar (SAR), information fusion, automatic target recognition (ATR), national imagery interpretability rating scale (NIIRS).

\section{Introduction}

High confidence combat identification (CID) is important to military operations to determine where friendly, enemy, and neutral combatants are located on the battle field. The goal of CID is to reduce fratricide and collateral damage to civilians while making it difficult for enemy targets to hide among friendly and neutral populations. Architectures employing cooperative and non-cooperative sensors have great potential to improve combat identification technologies and capabilities. However; sensors can be adversely affected by environmental factors such as weather and time of day, imaging geometries which obscure the target of interest, and/or enemy counter measures. Sensor, target, and environmental variations make robust CID difficult [1].

Many recognition algorithms have been proposed and performance results published in the literature to address the combat identification problem for both moving and stationary targets. The majority of the identification results have concentrated on single sensor measured and synthetic EO, IR, and SAR data $[2,3,4,5,6,7]$. Much of this work focused on a single sensor look at an area of interest to produce object identification decisions from one dimensional (1-D) signatures and two dimensional (2-D) images [8]. To improve upon single sensor look identification performance, CID algorithms fused multiple looks from the same sensor over different viewing geometries and times, exploiting additional target information gained from changes in the sensor-target geometry. Multi-look fusion can be accomplished a number of ways $[9,10]$, such as using a decision-level fusion algorithm [11] to combine single look recognition results in a serial fashion or by averaging 1-D signatures from all looks to create a mean signature that is then run through an ATR algorithm [12].

More recently, other sensor modalities, including hyperspectral (HSI), multispectral (MSI) [13, 14], laser radar (LADAR) [15, 16], and acoustic have been applied to the CID problem with some results appearing in the literature. Fusing features from different sensor geometries requires precise data registration as in [17] for HSI and SAR data because registration errors will adversely impact ATR decisions. The variable nature of different sensor modalities such as data format, operational sensitivities, and available features for fusion along with the issues that arise with registration of this data must be understood for successful demonstration of feature-level fusion algorithms.

To determine the best near-term feature fusion opportunities, the strengths, weaknesses, and technology maturity for six general categories of sensing systems were considered for potential CID performance gain. The sensing architectures investigated are SAR, LADAR, Spectral (MSI/HSI), EO, IR, and Vibrometry. Table 1 shows the general strengths and weaknesses for each category with the background color indicating the relative maturity (high maturity, moderate maturity, low maturity) of the sensor technology and data processing algorithms that include feature extraction and available ATR's. The authors acknowledge some sensor systems are more developed than others in any given category and table 1 is intended to represent the overlying technology and not any particular sensor system or development program. 


\begin{tabular}{|l|l|l|}
\hline $\begin{array}{c}\text { Sensor } \\
\text { Category }\end{array}$ & \multicolumn{1}{|c|}{ Strength } & \multicolumn{1}{|c|}{ Weakness } \\
\hline SAR & $\begin{array}{l}\text { Long range, 2-D/3-D imaging, } \\
\text { day/night, weather insensitive }\end{array}$ & Active sensing \\
\hline LADAR & $\begin{array}{l}\text { 2-D/3-D imaging, } \\
\text { day/night operation }\end{array}$ & $\begin{array}{l}\text { Active sensing, sensitive to } \\
\text { weather, sensitive to range }\end{array}$ \\
\hline $\begin{array}{l}\text { Passive sensing, material } \\
\text { identification, feature extraction } \\
\text { algorithms available }\end{array}$ & $\begin{array}{l}\text { Sensitive to weather, } \\
\text { Sensitive to range, } \\
\text { Day operation only for some } \\
\text { systems }\end{array}$ \\
\hline Electro-optic & $\begin{array}{l}\text { Passive sensing day, color } \\
\text { capable, motion video capable, } \\
\text { feature extraction algorithms } \\
\text { available }\end{array}$ & $\begin{array}{l}\text { Sensitive to time of day, } \\
\text { range dependant, sensitive to } \\
\text { weather }\end{array}$ \\
\hline Infrared & $\begin{array}{l}\text { Passive sensing day/night, } \\
\text { motion video capable, } \\
\text { temperature, feature extraction } \\
\text { algorithms available }\end{array}$ & $\begin{array}{l}\text { Range dependant, } \\
\text { Sensitive to weather }\end{array}$ \\
\hline Vibrometry & $\begin{array}{l}\text { Passive acoustic sensing, } \\
\text { day/night operation }\end{array}$ & $\begin{array}{l}\text { 1-D signature for 2-D } \\
\text { location, low resolution, } \\
\text { active LADAR sensing }\end{array}$ \\
\hline
\end{tabular}

Table 1. Sensor Phenomenology for Feature Level Fusion

LADAR, acoustical vibrometry, and MSI/HSI sensors provide a rich feature space for fusion exploitation; however, these technologies are still maturing and the fusion payoff will not be fully realized for a decade or more. Radar and electro-optical sensing technology is widely available and currently in use around the world for both military and civilian applications. An example of some of the military platforms and sensor modes $[18,19,20]$ in use are shown in Figure $\mathbf{1}$ along with representative sensor coverage areas for each system. A number of feature extraction and identification algorithms exist for use with EO/IR/SAR sensor data, leading to the conclusion that the greatest near-term high confidence identification performance potentially achievable is with EO/IR/SAR feature fusion.

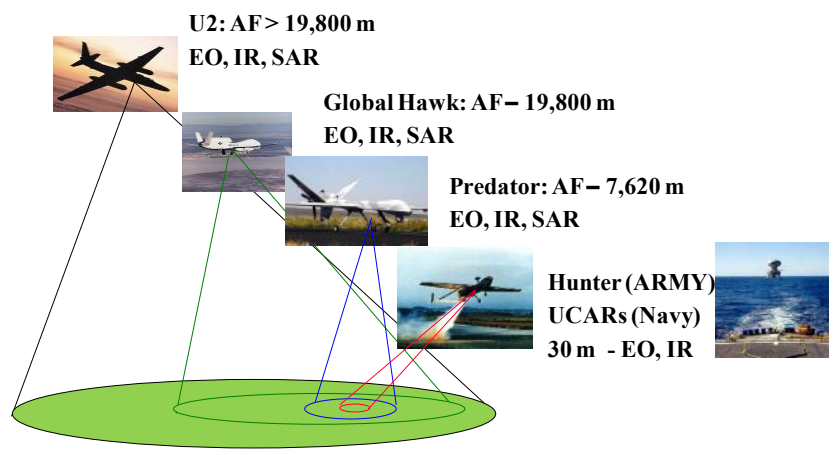

Figure 1. Example platforms, sensors, and coverage area

The selected scenario to study potential CID gains from the feature fusion of EO/IR/SAR sensors is a surveillance mission using EO/IR video and SAR stationary target identification.

The selection of EO, IR, and SAR sensor technology based on level of maturity allowed for the development of a fusion performance model that drew from the vast amount of performance prediction and modeling work applied to CID problems published in the available literature. The sections that follow will detail the key components of the fusion model, predict expected CID task discrimination results and fusion gains for each scenario, and discuss results and conclusions from the study.

\section{Feature Level Fusion Model}

An EO/IR/SAR fusion performance model was created to examine the impact of feature fusion to combat identification (CID) decisions and to assess the fusion gain potential for the modeled sensors. A single sensor, single look task discrimination model was created first to determine a CID performance baseline for each sensor mode selected. A feature fusion ID performance model was then developed to predict CID results. Task discrimination performance for both single sensor and fused feature models were compared to published results for verification.

\subsection{Feature Quality}

Feature-level fusion algorithms need to account for data quality. The quality of the data affects the value and number of available features that can be extracted for CID applications. Target features in poor quality imagery are increasingly merged together or obscured as quality degrades, rendering feature extraction difficult and adversely impacting CID decisions. An image quality model was developed using the national imagery interpretability rating scale (NIIRS) to assess the quality of EO/IR/SAR data. A description of the rating scale is found in Table 2 with estimated resolutions [21, 22, 23] included.

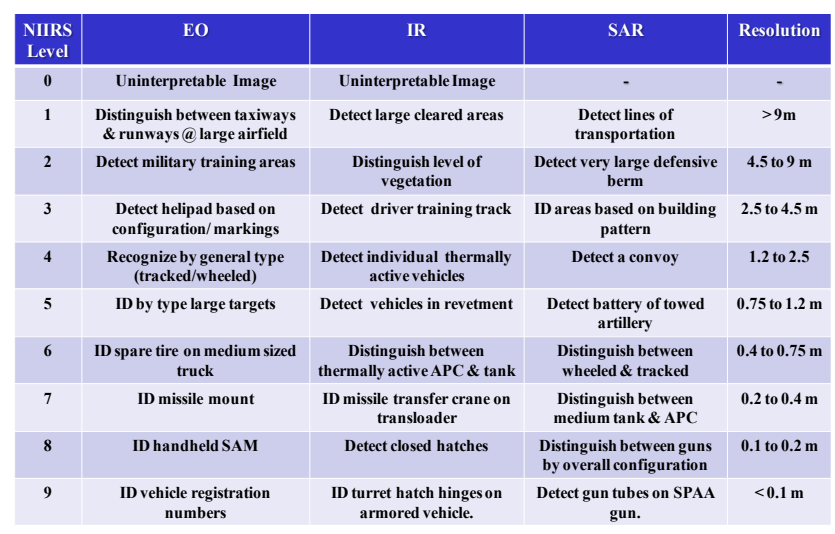

Table 2. NIIRS Definitions

General image quality equations (GIQE) were developed to predict NIIRS values based on sensor parameters. The general image quality equations for EO and IR sensors were implemented and related to a SAR sensor. The equation for an EO sensor [21] is given by

$$
\begin{aligned}
\text { NIIRS }_{\mathrm{EO}} & =10.251-a \log _{10}(G S D)+b \log _{10}(R E R) \\
& -0.656 H-[0.344(G / S N R)]
\end{aligned}
$$

where $G S D$ is the geometric mean of the ground sample distance, $H$ is the geometric mean height due to edge overshoot, RER is the geometric mean of the normalized relative edge response, $G$ is the noise gain, $S N R$ is the 
signal to noise ratio, $a$ is constant (3.32 if $R E R=0.9,3.16$ if $R E R<0.9$ ), and $b$ is constant ( 1.559 if $R E R=0.9,2.817$ if $R E R<0.9$ ). The variables and constant values remain the same for the infrared GIQE [21] given by the following expression

$$
\begin{aligned}
\text { NIIRS }_{\text {IR }} & =10.751-a \log _{10}(G S D)+b \log _{10}(R E R) \\
& -0.656 H-[0.344(G / S N R)]
\end{aligned}
$$

The SAR NIIRS is related to the IR NIIRS using the following equation developed in [21].

$$
\text { NIIRS }_{\text {IR }}=1.14+0.18 \mathrm{NIIRS}_{\mathrm{SAR}}+0.08 \mathrm{NIIRS}_{\text {SAR }}^{2} \text {. }
$$

The SAR resolution [21] is then determined by

$$
G S D=10^{\left[\left(10.751-\mathrm{NIIRS}_{\mathrm{IR}}\right) / a\right]}
$$

Where $a$ is the constant value from the GIQE and NIIRS is determined by Equation 3. The number of cycles across the target of interest is then given by

$$
\mathrm{N}=D c /(2 \bullet \mathrm{GSD})
$$

Where $D c$ is the critical target dimension given by

$$
D c=(l \bullet w \bullet h)^{(1 / 3)}
$$

and $l$ is target length, $w$ is target width, and $h$ is target height. The image quality results can then be related to the target discrimination tasks: detection, recognition, and identification using the target transfer probability function $[21,24,25]$ given in Equation 7.

$$
\mathrm{P}(\mathrm{N})=\left(\mathrm{N} / \mathrm{N}_{50}\right)^{\mathrm{a}+\mathrm{b}(\mathrm{N} / \mathrm{N} 50)} /\left[1+\left(\mathrm{N} / \mathrm{N}_{50}\right)^{\mathrm{a}+\mathrm{b}(\mathrm{N} / \mathrm{N} 50)}\right]
$$

where $\mathrm{a}$ and $\mathrm{b}$ are constants 1.75 and 0.35 respectively and $\mathrm{N}_{50}$ is the $\mathrm{N}$ value for $50 \%$ probability of success for a given discrimination task.

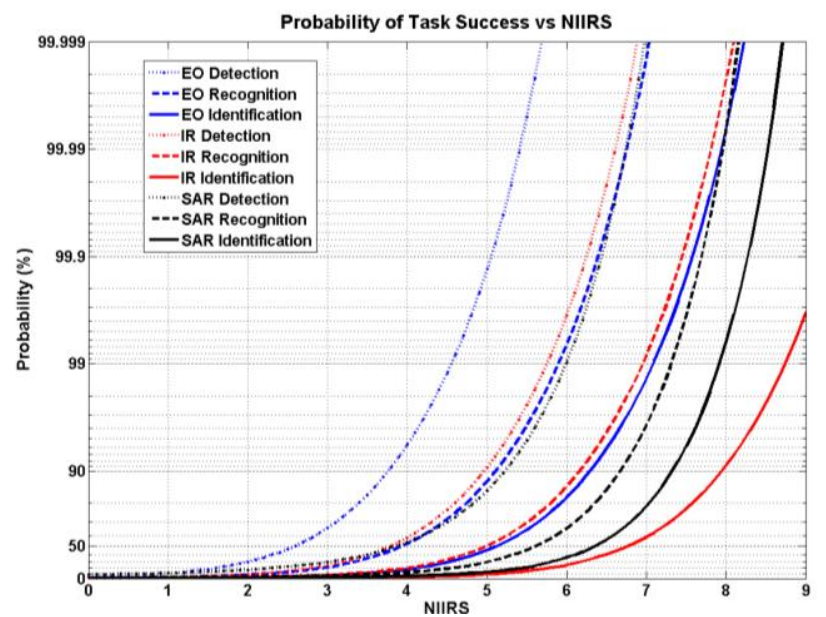

Figure 2. Sensor task discrimination vs. image quality

The probability of task success for EO, IR, and SAR sensors for a $6.6 \times 3.3 \times 3.4$ meter sized target are found in Figure 2. The curves illustrate the unique sensitivities of each sensor based on the various NIIRS levels necessary for successful task discrimination. Figure 2 indicates the feature independence between EO/IR/SAR imagery.

\subsection{Resolution and Range}

Next we relate the NIIRS values to sensor resolution by using Equations 1-4 and varying the NIIRS values between 0 and 9 to determine the GSD. The results are found in Figure 3.

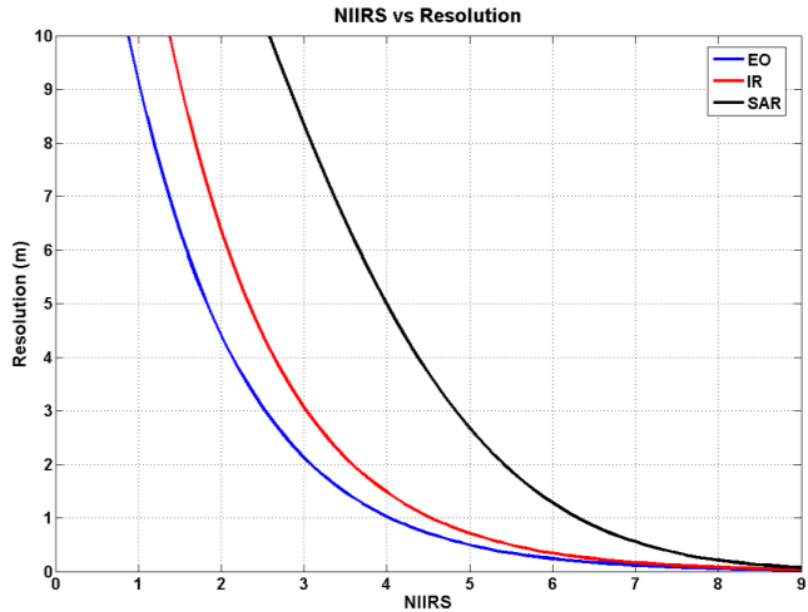

Figure 3. Image quality vs. sensor resolution

Then the sensor resolution is related to range for each sensor modality. Optical sensor resolution can be related to operational range by writing the $G S D$ in terms of sensor parameters as shown in Equation 8 where $F O V$ is the sensor field of view in degrees, $P$ is the number of pixels in the largest sensor dimension, and $R$ is the sensor range to target in meters.

$$
G S D=(F O V / P) R
$$

SAR resolution is a function of sensor parameters only and is therefore independent of range. The SAR cross range resolution is given by Equation 9 and the range resolution is found in Equation 10 where $\lambda$ is the wavelength, $L$ is the synthetic aperture extent, $B W$ is the radar bandwidth, and $c$ is the speed of light.

$$
\text { Cross Range Resolution }=\lambda / 2 L
$$

$$
\text { Range Resolution }=c / 2 B W
$$

The range sensitivity for optical sensors expressed in Equation 8 indicates that resolution improves at closer ranges and degrades at standoff sensing ranges. SAR sensor resolution is unaffected by range, making SAR an ideal standoff sensor. Figure 3 confirms better resolution increases image quality which in turn improves discrimination task performance as shown in Figure 2 for EO/IR/SAR sensors.

\subsection{Single Look Task Discrimination Model}

A single sensor, single look, task discrimination model was created using the target transfer probability function (TTPF) described in Equation 7 to determine baseline CID performance versus range for each sensor selected for a common target. Presented in Table 3 is a list of the 
sensor parameter values used for prediction of task success for each sensor. Equation 11 was used to determine the $\mathrm{N}_{50}$ [26] values of a given sensor for target detection.

$$
\mathrm{N}_{50}=0.75 C\left[(C / \Delta t)^{2}+1\right]
$$

where $C$ represents the image complexity of the data: $1-$ low, 1.5 - medium low, 2 - medium, and 2.7- high. $\Delta t$ is a constant ranging from 1 to 10 .

\begin{tabular}{|c|c|c|c|}
\hline Sensor Parameter & EO & IR & SAR \\
\hline FOV & $2.25^{\circ}$ & $1.5^{\circ}$ & N/A \\
\hline $\begin{array}{c}\text { Array Size } \\
\begin{array}{c}\text { Nominal Critical Target } \\
\text { Dimension }\end{array}\end{array}$ & $640 \times 480$ & $640 \times 480$ & N/A \\
\hline
\end{tabular}

Table 3. Sensor Parameters for task discrimination

Using Equations 5 - 8 for the same target and varying the range from 0 to $50 \mathrm{~km}$, the task discrimination performance is presented in Figure 4 for EO/IR/SAR sensors versus range to target. The SAR discrimination tasks show a gradual performance improvement at closer ranges that are caused by improved target-to-clutter ratios and not changes in resolution.

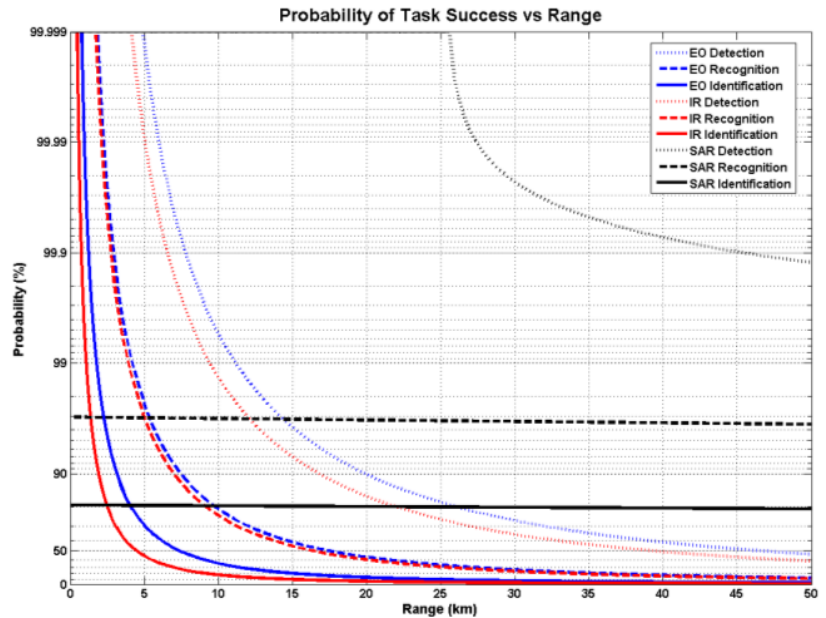

Figure 4. Sensor task discrimination vs. range

The optimal operation range selection for the chosen sensor technologies is possible from Figure $\mathbf{4}$ for achieving high probability of success for all three sensor modes.

\subsection{Fusion Definitions}

A fusion model detailing the primary functions, relevant information, databases, and interconnectivity for the performance of data fusion was developed and refined in the late 1980's and early 1990's by the Joint Directors of Laboratories' (JDL) Data Fusion Subpanel of the Technology Panel for C3 which contained levels 0 - 4 [27]. The JDL model was refined over the years $[28,29,30]$ and an additional level was added, User Refinement, as shown in Figure 5. The Data Information Fusion Group (DFIG) replaced the JDL model with a similar level 0-5 fusion model.

Tasks typically performed on a sensor by sensor basis such as measurement, signal processing, and filtering of the data fall under level 0 of the various fusion models. Tasks commonly associated with CID operations such as object detection, tracking, identification, group association, and intent fall in levels $1-3$. The higher fusion model levels 4 and 5 (JDL \& DFIG) are for optimization tasks such as mission planning, sensor placement, and algorithm selection.

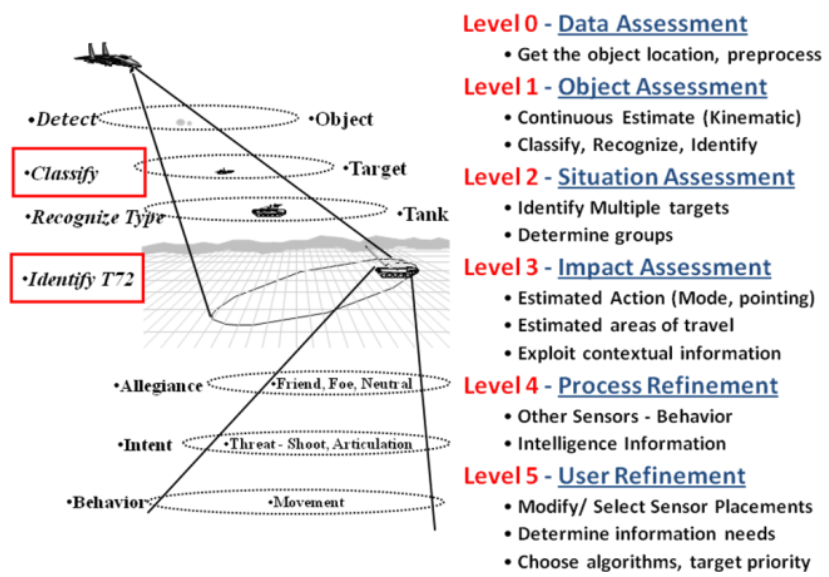

Figure 5. JDL Fusion Model and CID application

Object assessment is conducted at level 1 of the fusion models to determine when, where, and what an object is through data alignment, association, tracking, and identification [27]. Alignment projects the data into a common reference frame where data for an object of interest is associated via sorting or correlating observations so that estimating position and velocity is possible and object identification may occur. The types of level 1 fusion are image fusion, feature fusion, and decision fusion.

Decision Level Fusion (DLF) is the simplest fusion technique to implement but requires each sensor modality to have a good object recognition algorithm for classification. The DLF combines individual classifier outputs to form a fused identification decision.

Feature Level Fusion (FLF) is more complex than DLF to implement, requiring greater computational power. However, with increased complexity comes the potential for enhanced CID performance. The FLF combines the features of objects detected and segmented from each sensor domain. The independently detected object features need to be registered spatially and temporally before input to the FLF algorithm. Determining the appropriate features from each sensor modality is critical to FLF identification success.

Image Level Fusion (ILF) which is also known as pixel level fusion is the most complicated technique to implement and requires significant computational power. Registered pixel data from each sensor image is combined into a common reference frame for detection, recognition, 
and identification. Although this approach offers the greatest potential CID performance boost, sensitivity to registration errors will adversely impact ILF performance. Sensors imaging in different planes, measuring different phenomenologies, and in differing resolutions will likely require resampling and warping.

Many recent publications [11, 31] have explored the benefits of DLF to CID. The use of FLF algorithms is expected to further enhance CID performance beyond that obtained by using DLF technology. The optimal mix of features [32] must be determined for FLF to provide high confidence CID. Identified in Table 4 are some of the potential features that can be used for level 1 object assessment found in EO/IR/SAR sensor imagery.

\begin{tabular}{|c|c|c|}
\hline Category & Features & Utility \\
\hline $\begin{array}{l}\text { Geometric: } \\
\text { SAR \& EO }\end{array}$ & $\begin{array}{l}\text { Edges, lines, arcs, line } \\
\text { relationships }\end{array}$ & $\begin{array}{l}\text { Size, shape, structure, } \\
\text { kinematics of objects, } \\
\text { Pose Estimation }\end{array}$ \\
\hline $\begin{array}{l}\text { Structural: } \\
\text { SAR }\end{array}$ & $\begin{array}{l}\text { Number of surfaces, } \\
\text { orientation, position of } \\
\text { cylinders, cones, boxes }\end{array}$ & Pose \& configuration \\
\hline $\begin{array}{l}\text { EO/IR } \\
\text { Video }\end{array}$ & $\begin{array}{l}\text { Edges, texture, correlation } \\
\text { length, fractal dimension, } \\
\text { shape, aspect ratio, spatial } \\
\text { frequency distributions, } \\
\text { shadows }\end{array}$ & $\begin{array}{l}\text { Target class, coarse } \\
\text { level ID, \& kinematics }\end{array}$ \\
\hline SAR & $\begin{array}{l}\text { Grayscale, peaks, ridges, } \\
\text { edges, contours, shadows, } \\
\text { detection statistic aspect }\end{array}$ & $\begin{array}{l}\text { Strong discriminators } \\
\text { of components \& } \\
\text { structure }\end{array}$ \\
\hline $\mathbf{I R}$ & $\begin{array}{l}\text { Edges, regions, texture, } \\
\text { temperature spatial } \\
\text { distribution }\end{array}$ & $\begin{array}{l}\text { Unique thermal } \\
\text { signature \& } \\
\text { distribution }\end{array}$ \\
\hline
\end{tabular}

Table 4. Potential Features for CID

\subsection{Feature Fusion Performance Model}

A performance model was developed to predict the feature fused CID results and determine the fusion gain with respect to the best single sensor, single look recognition results. CID performance is often reported in a confusion matrix (CM) where each test target is compared against a library target and the recognition results tabulated in the $\mathrm{CM}$ with correct identification for each target along the diagonal of the CM. The FLF model was developed to predict the expected mean CID performance or report the mean of all correct target identities.

The predicted feature level fusion probability of correct identification is defined by Equation 12.

$$
\mathrm{FLF}_{\text {id }}=F_{\mathrm{a}} R \mathrm{e}^{(-1 /[1+\Sigma\{9 P \mathrm{id} / \mathrm{NIIRS}\}])}
$$

where $P_{\text {id }}$ is the mean probability of correct identification for all targets from a given sensor, NIIRS is the image quality for each sensor, $F_{\mathrm{a}}$ is the appropriateness of the features being fused $(0-$ poor through $1-$ optimum $)$, and $R$ is the feature registration accuracy $(0-$ no registration through 1 - perfect registration).

The $P_{\text {id }}$ and NIIRS parameters used in Equation 12 are obtained from the image quality and resolution equations used to generate Figures 2-4. If the features from three sensors are to be fused, using the probability of correct identification from each sensor the corresponding NIIRS values could be obtained from Figure 2. These values are then put into Equation 12 along with the desired registration and feature optimization percentages to obtain a predicted FLF ID.

Once the predicted fused CID result is known, the feature fusion gain with respect to the best single sensor CID performance is determined using Equation 13.

$$
\text { Fusion Gain }=\left(\mathrm{FLF}_{\mathrm{id}}-\mathrm{SSSL}_{\mathrm{id}}\right) / \mathrm{SSSL}_{\mathrm{id}}
$$

where $\mathrm{FLF}_{\mathrm{id}}$ is the probability of correct identification obtained from feature level fusion, $\mathrm{SSSL}_{\text {id }}$ is the best single sensor single look probability of correct identification regardless of sensor mode.

\subsection{Fusion Model Validation}

The FLF CID performance model was validated against automatic target recognition (ATR) results using measured $\mathrm{EO} / \mathrm{IR} / \mathrm{SAR}$ sensor data found in the literature. A series of experiments were conducted and the model predictions compared to published data to validate point solutions in the decision space.

As discussed earlier, feature fusion presumes independent detection in each sensor and combines the extracted features in a common decision space. FLF is similar to multi-look recognition algorithms which use sensor data that is closely associated in space and time to achieve enhanced identification performance. Both multi-look and FLF CID algorithms are optimized for classification of selected targets. For a single sensor, the FLF model should produce results which correspond to single sensor multi-look ATR performance.

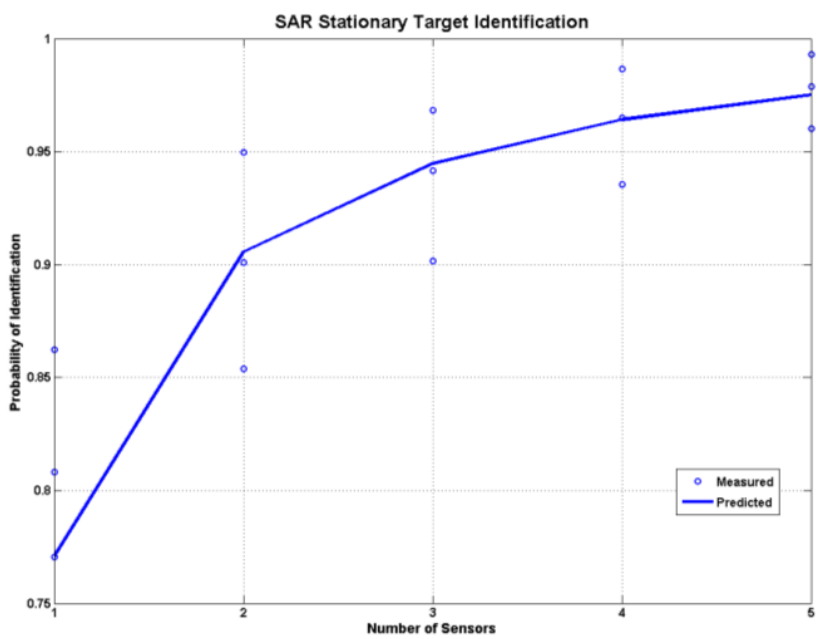

Figure 6. SAR Stationary Target Identification

In a recent paper [9] investigating multi-look classification using collected SAR data of stationary targets, three ATR algorithms (K-nearest neighbor, Bayesian, and Feed-forward artificial neural network) were used to generate classification results to alleviate any algorithm bias. Average classification performance was reported for each algorithm as the number of looks 
increased. The fusion performance model treated each new look as a different sensor and predicted the fused CID. In Figure 6 the expected FLF identification is compared to the ATR performance of all three algorithms versus the number of looks. The fusion model from Figure 6 shows good agreement with the published data. The fusion model used optimal features and ideal feature registration to produce the results.

Choosing the optimum mix of features for fusion will impact CID performance which is why the FLF model includes a feature selection parameter. The effect of selecting the wrong features or too many features was the topic of a feature based single-look SAR ATR investigation $[33,34]$. The average ATR performance for stationary targets versus a set of features is presented in Figure 7 and compared to the FLF performance model. The FLF model had constant NIIRS values, ideal registration, and constant probability of identification, leaving only the feature parameter to be varied.

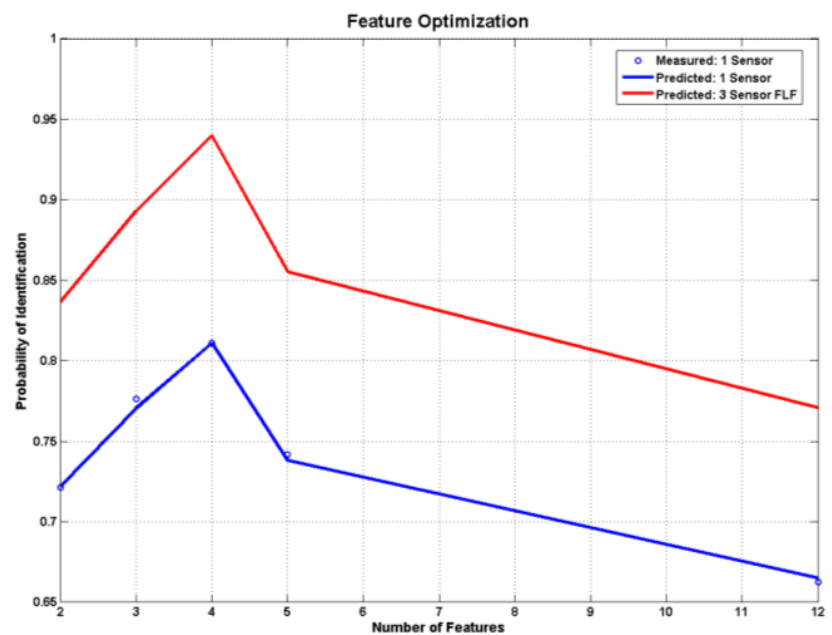

Figure 7. SAR Feature Optimization

The FLF model validation efforts have so far relied on SAR performance data. Published data fusion results for $\mathrm{EO} / \mathrm{IR}$ [35], IR[31], and IR/SAR[36] data is presented in Table 5 along with predict FLF performance. Ideal registration and optimal features were selected for all of the predicted FLF identification. The IR data only fusion results presented are for the mean of six DLF algorithms and the IR probability of identification reported in the Table 5 is the average of four single look ATR algorithms used for the DLF performance comparison. The FLF model agrees with the published feature level fusion performance and exceeds the mean DLF performance.

\begin{tabular}{|c|c|c|c|c|}
\hline \multicolumn{3}{|c|}{ Pid } & \multicolumn{2}{c|}{ FLF } \\
\hline EO & IR & SAR & Reported & Predicted \\
\hline $\mathbf{0 . 8 8}$ & $\mathbf{0 . 8 5}$ & N/A & $\mathbf{0 . 9 1}$ & $\mathbf{0 . 9 2}$ \\
\hline N/A & $\mathbf{0 . 7 5}$ & N/A & 0.81 & 0.95 \\
\hline N/A & $\mathbf{0 . 5 8}$ & $\mathbf{0 . 4 0}$ & 0.78 & 0.83 \\
\hline
\end{tabular}

Table 5. Predicted vs. Reported FLF Performance

\section{Scenario}

An intelligence, surveillance, and reconnaissance (ISR) type mission was selected for feature fusion analysis using a combination of electro-optical, infrared, and synthetic aperture radar sensors for target identification. Each sensor images the same area and mix of targets for stationary target identification using EO/IR video and SAR imagery

A combination of sensors and potential target scenarios is graphically depicted in Figure 8. The overlap in the center of Figure $\mathbf{8}$ of the sensors and target conditions is the subject of a recent paper [37] discussing the GOTCHA Radar and NightStare development programs at the Air Force Research Laboratory (AFRL). The colored sections and dots in the center region show potential fusion opportunities for enhanced CID.

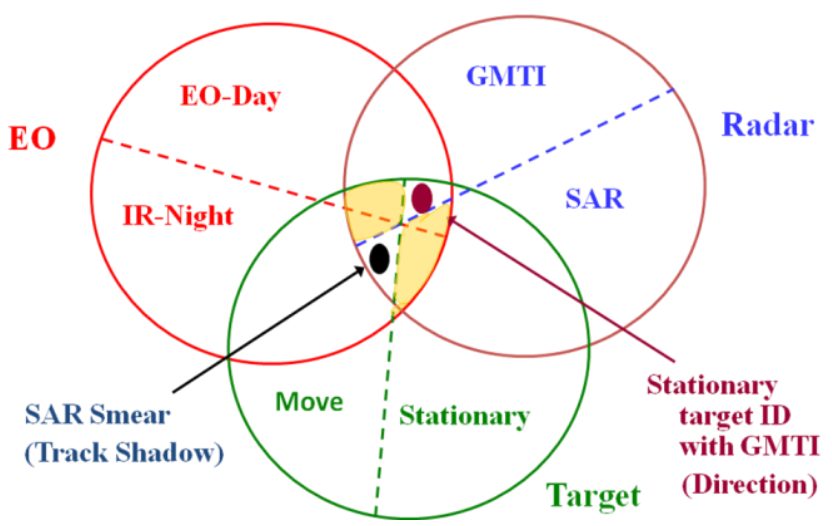

Figure 8. Sensor-Scenario Combinations

The use of unmanned air vehicles (UAVs) in military operations especially in hostile environments has increased dramatically. EO/IR sensors are commonly found on many of these UAV systems. SAR sensors are typically employed on standoff platforms because radars are range invariant. Fusing the features of forward deployed EO/IR sensors with those of standoff SAR sensors should provide high confidence CID of stationary high value targets. Registration errors of the extracted features from multiple geometries will impact FLF ID performance but could be mitigated by combining the EO/IR video sensors with the SAR sensor on a single platform.

\begin{tabular}{|c|c|}
\hline Parameter & Value \\
\hline EO P $_{\text {id }}$ & $\mathbf{0 . 7 0}$ \\
\hline EO NIIRS & $\mathbf{5 . 6}$ \\
\hline IR P $_{\text {id }}$ & $\mathbf{0 . 6 0}$ \\
\hline IR NIIRS & 7 \\
\hline SAR P $_{\text {id }}$ & $\mathbf{0 . 7 0}$ \\
\hline SAR NIIRS & $\mathbf{6 . 8}$ \\
\hline Feature Registration & $0-1$ \\
\hline Feature Optimization & $\mathbf{0 . 2 5 , 1 . 0}$ \\
\hline
\end{tabular}

Table 6. Fusion model parameters 
The parameters that were used to predict FLF identification performance for stationary target identification are found in Table 6. The sensor collection geometries were all different leading to variable NIIRS and $P_{\text {id }}$ values. Registration and optimal features are varied to produce upper and lower performance bounds.

\section{Results}

Feature level fusion performance was predicted for each scenario defined in the previous section using the parameters identified in Table 6. The FLF model was used to predict expected performance limits by varying the available features and corresponding feature registration. The results of the ISR scenario are found in Figure 9.

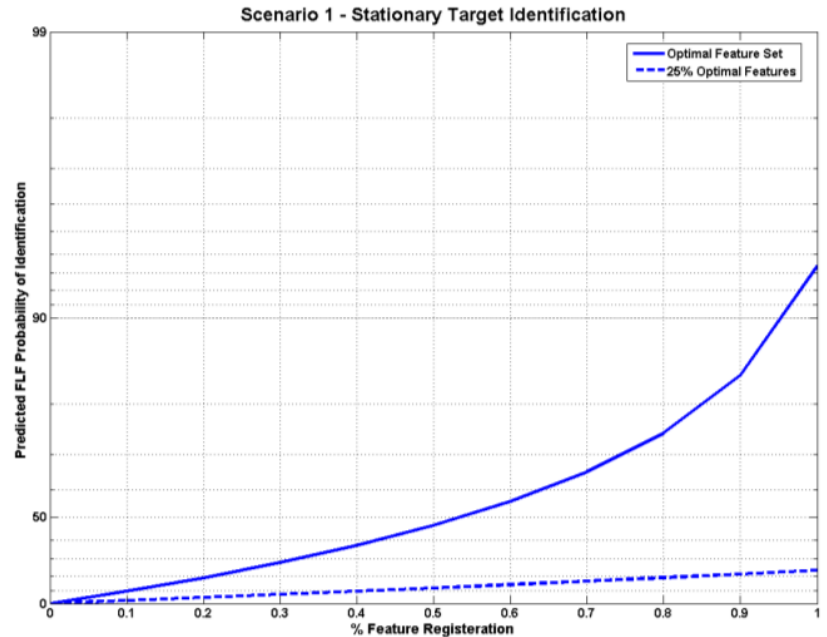

Figure 9. Predicted FLF Stationary Target ID Performance

The predicted FLF performance is related to the best single sensor identification performance for each scenario to determine relative fusion gain or loss using Equation 13. A significant improvement in CID performance is shown for the stationary target scenario in Table 7 with a $33.41 \%$ increase in object identification.

\begin{tabular}{|l|c|}
\hline \multicolumn{1}{|c|}{ Description } & Value \\
\hline Best Sensor $\mathbf{P}_{\text {id }}$ & 0.70 \\
\hline FLF P $_{\text {id }}$ & 0.93 \\
\hline Fusion Gain & 0.33 \\
\hline
\end{tabular}

Table 7. EO/IR/SAR Stationary Target ID Fusion Gain

\section{Conclusion}

A feature fusion model was developed by first creating task discrimination models for EO/IR/SAR sensors which take into account key factors for object identification such as sensor resolution, image quality, and range to target. Estimated detection, recognition, and identification curves were created for each sensor for a nominal sized target versus the key factors listed previously. The output from the task discrimination model was input into a feature level fusion target identification model which related the probability of correct identification to image quality on a sensor by sensor basis and accounted for the appropriateness of the features and how well the features were registered. Finally, an expected fusion gain was determined by relating the predicted FLF ID to the best single look, single sensor ID. Stationary target identification achieved a significant performance increase, $33.41 \%$.

To verify the prediction behavior of the FLF performance model, mean identification performance values were determined from published multi-look ATR results using measured data for comparison. A few examples of FLF target identification using EO/IR/SAR sensors were located in the literature as another comparison point. The model showed good agreement with all of the published data.

Predicted feature fusion performance from multiple sensors of diverse phenomenology and viewing geometries has been demonstrated to improve target identification performance over single sensor CID. Expected FLF performance bounds were determined that took into account the importance of using optimal features for identification and how well the selected features were registered to one another. Feature level fusion using the optimal combination of target features, kinematic and mission data significantly enhances CID performance relative to single look sensor recognition.

\section{References}

[1] B. Kahler, E. Blasch, L. Goodwon, "Operating Condition Modeling for ATR Fusion Assessment", Multisensor, Multisource Information Fusion, Proc. Of SPIE, Vol. 6571, April 2007.

[2] E. Blasch and B. Kahler, "Multiresolution EO/IR Target Tracking and Identification", IEEE Proc. Of the $8^{\text {th }}$ International Conference On Information Fusion, July 2005.

[3] B. Weber and J. Penn, "Improved Target Identification Using Synthetic Infrared Images", Automatic Target Recognition XII, Proc. Of SPIE, Vol. 4726, 2002.

[4] B. Correia and R. Nunes, "Grouping multiple neural networks for automatic target recognition in infrared imagery", Automatic Target Recognition XI, Proc. Of SPIE, 2001.

[5] R. Schumacher and J. Schiller, "Non-cooperative Target Identification of Battlefield Targets-Classification Results Based on SAR Images", IEEE, 2005.

[6] R. Williams, J. Westerkamp, D. Gross, and A. Palomino, "Automatic Target Recognition of Time Critical Moving Targets Using 1D High Range Resolution (HRR) Radar", IEEE AES Systems Magazine, April 2000.

[7] B. O'Kane, I. Biederman, and E. Cooper, "Modeling Parameters For Target Identification: A Critical Features Analysis", Proceedings of the IRIS Passive Sensors Symposium, March 1996.

[8] L. Horowitz, G. Brendel, "Fundamental SAR ATR Performance Predictions for Design Tradeoffs: 1-D HRR versus 2-D SAR versus 3-D SAR", Algorithms for Synthetic Aperture Radar Imagery VI, Proc. Of SPIE, 1999.

[9] M. Vespe, C. Baker, and H. Griffiths, "Radar target classification using multiple perspectives", IET Radar Sonar Navig., Vol. 1, No. 4, August 2007.

[10] A. Paul, A. Shaw, K. Das, and A. Mitra, "Improved HRR-ATR using Hybridization of HMM and Eigen-Template-Matched Filtering”, IEEE International Conference on Acoustics, Speech, and Signal Processing, Vol. 2, April 2003. 
[11] B. Kahler and E. Blasch, "Robust Multi-Look HRR ATR Investigation Through Decision-Level Fusion Evaluation", IEEE $11^{\text {th }}$ International Conference On Information Fusion, July 2008.

[12] B. Kahler and A. Palomino, "Simulation of UAV Sensor System Parameter Effects on High Range Resolution ATR Performance", Nation Fire Control Symposium, July 2003.

[13] J. Kerekes and S. Hsu, "SpectralQuality Metrics for VNIR and SWIR Hyperspectral Imagery", Algorithms and Technologies for Multispectral, Hyperspectral, and Ultraspectral Imagery X, Proc. Of SPIE, Vol. 5425, 2004.

[14] J. Kerekes, A. Cisz, and R. Simmons, "A comparative evaluation of spectral quality metrics for hyperspectral imagery", Algorithms and Technologies for Multispectral, Hyperspectral, and Ultraspectral Imagery XI, Proc. Of SPIE, Vol. 5806, 2005.

[15] J. Khoury, C. Woods, J. Lorenzo, J. Kierstead, D. Pyburn, and S. Sengupta, "Resolution limits in imaging LADAR systems", Applied Optics, Vol. 45, No. 4, February 2006.

[16] P. Andersson, "Long-range three-dimensional imaging using rangegated laser radar images", Optical Engineering, Vol. 45, No. 3, March 2006.

[17] H. Szu and C. Hsu, "Ultraspectral: Hyperspectral and RF Features Registered by IFSAR", Wavelet Applications VIII, Proc. Of SPIE, Vol. 4391, 2001.

[18] “U.S. Air Force, Air Force Roadmap 2006-2025”, June 2006.

[19] Department of Defense, "Unmanned Systems Roadmap 20072032", December 2007

[20] Office of the Secretary of Defense, "Unmanned Aircraft Systems Roadmap 2005 - 2030", August 2005.

[21] R. Driggers, J. Ratches, J. Leachtenauer, R. Kistner, "Synthetic aperture radar target acquisition model based on a National Imagery Interpretability Rating Scale to probability of discrimination conversion", Optical Engineering, Vol. 42, No. 7 , July 2003.

[22] R. Driggers, P. Cox, and M. Kelley, "National imagery interpretation rating system and the probabilities of detection, recognition, and identification", Optical Engineering, Vol. 36, No. 7, July 1997.

[23] R. Driggers, M. Kruer, D. Scribner, P. Warren, and J. Leachtenauer, "Sensor Performance Conversions for Infrared target acquisition and intelligence-surveillance-reconnaissance imaging sensors", Applied Optics, Vol. 38, No. 28, Oct. 1999.

[24] K. Krapels, R. Driggers, P. Larson, J. Garcia, B. Walden, S. Agheera, D. Deaver, J. Hixson, E. Boettcher, "Small Craft ID Criteria (N50/V50) for Short Wave Infrared Sensors in Maritime Security", Infrared Imaging Systems: Design, Analysis, Modeling, and Testing XIX, Proc. Of SPIE, Vol. 6941, 2008.

[25] J. Doe, E. Boettcher, and B. Miller, "Identification of ground targets from airborne platforms", Infrared Imaging Systems: Design, Analysis, Modeling, and Testing XX, Proc. Of SPIE, Vol. $7300,2009$.

[26] R. Driggers, E. Jacobs, R. Vollmerhausen, B. O’Kane, S. Moyer, J. Hixson, G. Page, K. Krapels, D. Dixon, R. Kistner, and J. Mazz, "Current Infrared Target Acquisition Approach for Military Sensor Design and Wargaming", Infrared Imaging Systems: Design, Analysis, Modeling, and Testing XVII, Proc. Of SPIE, Vol. 6207, 2006.

[27] F. E. White Jr., Data Fusion Lexicon, Joint Directors of Laboratories, Technical Panel for $\mathrm{C}^{3}$, Data Fusion Subpanel, Naval Ocean Systems Center, San Diego, 1987.

[28] D. L. Hall, Mathematical Techniques in Multisensory Data Fusion, Artech House, Inc., 1992.

[29] R. Mahler, "A Unified Foundation for Data Fusion", Proc. 1994 Data Fusion Systems Conference, Oct. 1994.

[30] A. N. Steinberg, C. L. Bowman, and F. E. White, "Revisions to the JDL Data Fusion Model", Sensor Fusion: Architectures, Algorithms, and Applications III, Proc. Of SPIE, Vol. 3719, April 1999.

[31] S. A. Rizvi, N.M. Nasrabadi, "Fusion of FLIR automatic target recognition algorithms", Information Fusion 4, Elsevier, 2003.
[32] T. M. Schuck, J. B. Hunter, and D. D. Wilson, "Multi-Hypothesis Structures and Taxonomies for Combat Identification Fusion", IEEE Aerospace Conference Proc., 2004.

[33] J. Liu, and K. Chang, "Feature-based target recognition with a Bayesian network", Optical Engineering, Vol. 35, No. 3, March 1996.

[34] P. Dhillon, D. Foster, and L. Ungar, "Efficient Feature Selection in the Presence of Multiple Feature Classes", IEEE Computer Society, Eighth IEEE International Conference on Data Mining, 2008.

[35] S. Giompapa, R. Croci, R. DiStefano, A. Farina, F. Gini, A. Graziano, and F. Lapierre, "Naval target classification by fusion of IR and EO sensors", Electro-optical and Infrared Systems: Technology and Applications IV, Proc. Of SPIE, Vol. 6737, 2007.

[36] J. Ratches, C. Walters, R. Buser, and B. Guenther, "Aided and Automatic Target Recognition Based Upon Sensory Inputs From Image Forming Systems", IEEE Transactions on Pattern Analysis And Machine Intelligence, Vol. 19, No. 9, September 1997.

[37] M. Bryant, "Multisensor Staring Exploitation", Proc. Of SPIE, Vol. $6981,2008$. 